\title{
Diffuse-interface modeling of liquid-vapor phase separation in a van der Waals fluid
}

\author{
A. G. Lamorgese ${ }^{1}$ and R. Mauri ${ }^{2}$ \\ ${ }^{1}$ Center for Turbulence Research, Stanford University, Stanford, California 94305-3035, USA \\ ${ }^{2}$ Department of Chemical Engineering, Industrial Chemistry and Material Science, Università di Pisa, \\ 56126 Pisa, Italy
}

(Received 15 September 2008; accepted 26 February 2009; published online 23 April 2009)

\begin{abstract}
We simulate liquid-vapor phase separation in a van der Waals fluid that is deeply quenched into the unstable range of its phase diagram. Our theoretical approach follows the diffuse-interface model, where convection induced by phase change is accounted for via a nonequilibrium (Korteweg) force expressing the tendency of the liquid-vapor system to minimize its free energy. Spinodal decomposition patterns for critical and off-critical van der Waals fluids are studied numerically, revealing the scaling laws of the characteristic length scale and composition of single-phase microdomains, together with their dependence on the Reynolds number. Unlike phase separation of viscous binary mixtures, here local equilibrium is reached almost immediately after single-phase domains start to form. In addition, as predicted by scaling laws, such domains grow in time like $t^{2 / 3}$. Comparison between 2D and 3D results reveals that 2D simulations capture, even quantitatively, the main features of the phenomenon. (C) 2009 American Institute of Physics.
\end{abstract}

[DOI: $10.1063 / 1.3103826$ ]

\section{INTRODUCTION}

We present simulations of isothermal liquid-vapor spinodal decomposition of a van der Waals fluid, occurring after quenching it from a single-phase equilibrium state to the unsteady two-phase region of its phase diagram, showing that the process is governed by the density gradient-driven nonequilibrium Korteweg body force.

Experimentally, most studies on spinodal decomposition have been performed using light-scattering techniques in microgravity. In fact, under normal gravity conditions, the density difference between the evolving phases in the two-phase fluid causes sedimentation and formation of layered structures, which makes growth measurements of single-phase microdomains hard to perform. That is why most experiments (conducted under normal gravity conditions) on spinodal decomposition have been carried out using nearly isopycnic binary liquids. There, the mixtures start to separate by diffusion and coalescence right after the temperature has crossed that of the miscibility curve, leading to the formation of dendritic, interconnected domains for critical systems, or pseudospherical drops of the minority phase surrounded by the majority phase for off-critical systems. In particular, it was shown ${ }^{1-5}$ that diffusion alone cannot explain the segregation process in low-viscosity liquid mixtures, as the late stage of phase separation is dominated by convection, leading to a linear growth law for the characteristic size of single-phase microdomains.

Although experiments and simulations on liquid-vapor phase separation have been carried out in the past, ${ }^{6-12}$ none of these previous works has focused on spinodal decomposition systematically. In this work, applying the diffuseinterface model, ${ }^{13-20}$ we investigate liquid-vapor spinodal decomposition in $2 \mathrm{D}$ and $3 \mathrm{D}$ for critical and off-critical van der
Waals fluids as a function of a convection parameter expressing the relative magnitude of capillary to viscous forces. We show that, at the late stages of the process, the mechanism of growth is convection-driven coalescence with a $2 / 3$ powerlaw scaling for the characteristic size of single-phase microdomains, in agreement with dimensional analysis ${ }^{21,22}$ and experimental measurements. ${ }^{9}$ Inertial scaling of convectiondriven coalescence has also been reported in lattice Boltzmann simulations of binary fluid spinodal decomposition. ${ }^{6,23-25}$ In particular, it has been proposed ${ }^{26,27}$ that the behavior in the inertial range is likely to be more complex than suggested by simple dimensional analysis. In fact, according to Kendon, ${ }^{27}$ multiple length scales become relevant, but this requires further investigation via higher resolution simulations.

The rest of this paper is organized as follows. In Sec. II we describe the diffuse-interface model, showing that momentum transport is enhanced by a nonequilibrium Korteweg force. Then, in Sec. III, our numerical method is briefly described and validated by simulating liquid droplet coalescence in vapor at equilibrium, comparing our predictions with well-known results. Finally, the spinodal decomposition patterns and the scaling law for the typical domain size in the viscous and inertial regimes are discussed. At the end, a few conclusions are drawn.

\section{THE GOVERNING EQUATIONS}

The diffuse-interface model for a pure single-component fluid is based on the van der Waals mean field theory of capillarity. ${ }^{28}$ In this theory, the effects of spatial inhomogeneities of the fluid density, $\rho$, are accounted for via a generalized Helmholtz free energy, given by 


$$
f(\rho, \nabla \rho)=f_{\text {th }}(\rho)+\Delta f_{n l}(\nabla \rho) .
$$

Here, $f_{\text {th }}$ gives the thermodynamic specific (i.e., per unit volume) free energy of a van der Waals fluid,

$$
f_{\text {th }}=\frac{\rho R T}{M_{W}} \log \frac{\rho}{1-c_{2} \rho}-c_{1} \rho^{2},
$$

where $c_{1}$ and $c_{2}$ are the pressure adding term and the excluded volume, respectively, $R$ is the gas constant, $T$ is the temperature, and $M_{W}$ is the molecular weight. As a consequence of the nonlocality of molecular interactions in the van der Waals mean field theory, the second term on the righthand side of Eq. (1) is a nonlocal specific free energy due to density changes, given by ${ }^{29,30}$

$$
\Delta f_{n l}=\frac{1}{2} \frac{R T a^{2}}{M_{W}} K(\nabla \rho)^{2},
$$

where $K=N_{A} d^{3} / M_{W}$ is a constant reciprocal density (of the same magnitude as the specific volume of the liquid phase), $N_{A}$ is the Avogadro number, $d$ is a molecular diameter, while $a$ is a typical distance describing the interface thickness. As shown by van der Waals, ${ }^{31}$ since the surface tension $\sigma$ is the energy stored in the unit area of the liquid-vapor interface at local equilibrium, we obtain

$$
\sigma=\frac{R T a^{2}}{M_{W}} K \int(\nabla \rho)^{2} d l \sim a \frac{R T}{M_{W}} K(\Delta \rho)_{\mathrm{eq}}^{2},
$$

where $(\Delta \rho)_{\text {eq }}$ is the density difference between the two phases at equilibrium. Therefore, the characteristic length $a$ can be determined once the surface tension is known.

Now, the equilibrium state of a nonhomogeneous van der Waals fluid is that which minimizes the functional $f$ [Eq. (1)], subjected to the constraint of mass conservation. For general, nonequilibrium conditions, conservation of mass and momentum (in the absence of external forces) lead to the following equations:

$$
\begin{aligned}
& \partial_{t} \rho+\nabla \cdot(\rho \mathbf{u})=0, \\
& \partial_{t}(\rho \mathbf{u})+\nabla \cdot(\rho \mathbf{u} \otimes \mathbf{u}+\boldsymbol{\Pi})=0,
\end{aligned}
$$

where $\mathbf{u}$ is the local fluid velocity and $\boldsymbol{\Pi}$ is the momentum flux density. These equations are normally coupled to the equation of energy conservation. However, since in this work we focus on isothermal processes, this last equation can be disregarded, as it is identically satisfied. The method of irreversible thermodynamics ${ }^{32}$ is then used for deriving an expression for $\Pi$, subject to the requirement that the reversible, nonequilibrium (Korteweg) stresses (due to the nonlocal component of the free energy) not contribute to the rate of entropy production. At the end, for Newtonian mixtures, we obtain $^{16}$

$$
\Pi=p \mathbf{I}+\Pi_{\rho}+\boldsymbol{\tau},
$$

where $p$ is the pressure, $\mathbf{I}$ is the identity tensor, while $\boldsymbol{\Pi}_{\rho}$ and $\tau$ denote the Korteweg and viscous stress tensors, respectively:

$$
\boldsymbol{\Pi}_{\rho}=\frac{R T a^{2}}{M_{W}} K\left[\nabla \rho \otimes \nabla \rho-\left(\rho \nabla^{2} \rho+\frac{1}{2}|\nabla \rho|^{2}\right) \mathbf{I}\right],
$$

$$
\boldsymbol{\tau}=-\eta\left[\nabla \mathbf{u}+\nabla \mathbf{u}^{T}-\frac{2}{3}(\nabla \cdot \mathbf{u}) \mathbf{I}\right],
$$

with $\eta$ denoting the dynamic viscosity which, in analogy with ideal mixtures, we assume to be linearly dependent on density. Accordingly,

$$
\widetilde{\eta} \equiv \frac{\eta}{\eta_{\mathrm{I}}}=r+(1-r) \frac{\rho-\rho_{\mathrm{II}}}{\rho_{\mathrm{I}}-\rho_{\mathrm{II}}},
$$

where $r=\eta_{\mathrm{II}} / \eta_{\mathrm{I}}$ denotes the viscosity ratio, with the subscript I and II denoting the liquid and vapor phases at equilibrium, respectively.

The Korteweg force appearing in the momentum equation, $\mathbf{F}_{\rho}=-\nabla \cdot \boldsymbol{\Pi}_{\rho}$, is a reversible body force, driven by density gradients in the fluid. In particular, at the late stages of phase separation, after the fluid has developed well-defined liquid/vapor domains separated by sharp interfaces, this body force reduces to the more conventional Marangoni force, as shown by Jasnow and Viñals ${ }^{17}$ and Jacqmin. ${ }^{33}$

The conservation equations (5) and (6) must be supplemented by an equation of state. From the expression (2) of the thermodynamic free energy, applying $p=\rho\left(\partial f_{\mathrm{th}} / \partial \rho\right)_{T}$ $-f_{\text {th }}$, we obtain the van der Waals equation of state:

$$
p=\frac{\rho R T / M_{W}}{1-c_{2} \rho}-c_{1} \rho^{2} .
$$

In this paper, we intend to study liquid-vapor spinodal decomposition by perturbing unstable van der Waals fluids with delocalized (random) density fluctuations. We also assume the characteristic single-phase domain size to be always much less than the macro-length-scale $L$, thus implying a negligible Bond number. Under these conditions, in the early, diffusion-driven stages of phase separation, it is natural to consider a diffusive scaling of the equations, defined by

$$
\begin{aligned}
& \widetilde{\mathbf{x}}=\frac{\mathbf{x}}{L}, \quad \tilde{t}=\frac{\eta_{\mathrm{I}} t}{\rho_{c} L^{2}}, \quad \widetilde{\mathbf{u}}=\frac{\mathbf{u} L \rho_{c}}{\mathcal{R} \eta_{\mathrm{I}}}, \\
& \mathcal{R}=\frac{\rho_{c}^{2} R T a^{2}}{M_{W} \eta_{\mathrm{I}}^{2}}, \quad \beta=\frac{N_{A} d^{3}}{M_{W}} \rho_{c}, \\
& \tilde{\rho}=\frac{\rho}{\rho_{c}}, \quad \tilde{p}=\frac{p}{\rho_{c} R T_{c} / M_{W}}, \quad \tilde{T}=\frac{T}{T_{c}} .
\end{aligned}
$$

Here, $\rho_{c}$ and $T_{c}$ are the critical density and temperature, and $p_{c}=\rho_{c} R T_{c} / M_{W}$ is the pressure at critical conditions if the fluid were a perfect gas, $\beta$ is an $O(1)$ density ratio, while the nondimensional parameter $\mathcal{R}$, defined as the ratio of capillary to viscous forces, represents an inverse capillary number. In fact, $\mathcal{R}$ also plays the role of an interfacial Reynolds number, $\mathcal{R}=\rho_{c} V_{r} a / \eta_{\mathrm{I}}$, based on a characteristic velocity which can be estimated from the Korteweg force as $V_{r}$ $\sim \rho_{c} R T a /\left(M_{W} \eta_{\mathrm{I}}\right)$.

In the late stages of the process, it is preferable to use an acoustic, i.e., convective, scaling instead of the diffusive scaling (12). Accordingly, defining a reference sound speed, $u_{s}=\left(R T_{c} / M_{W}\right)^{1 / 2}$, we set 


$$
\widetilde{\mathbf{x}}=\frac{\mathbf{x}}{L}, \quad \tilde{t}=\frac{u_{s} t}{L}, \quad \widetilde{\mathbf{u}}=\frac{\mathbf{u}}{u_{s}}, \quad \operatorname{Re}=\frac{\rho_{c} u_{s} L}{\eta_{\mathrm{I}}},
$$

while the nondimensional density, temperature, and pressure are defined as in Eq. (13). Also, Re is an acoustic Reynolds number which can be used to rewrite the interfacial Reynolds number as

$$
\mathcal{R}=(\operatorname{Re} C n)^{2}=\left(\frac{\rho_{c} u_{s} a}{\eta_{\mathrm{I}}}\right)^{2},
$$

where the Cahn number, $C n=a / L$, is the ratio of the interface thickness to the macro-length-scale $L$. Finally, the governing equations in dimensionless form (with all tildes dropped) based on the acoustic scaling read as follows:

$$
\begin{aligned}
& \partial_{t} \rho+\nabla \cdot(\rho \mathbf{u})=0, \\
& \partial_{t}(\rho \mathbf{u})+\nabla \cdot(\rho \mathbf{u} \otimes \mathbf{u})+\nabla p+\frac{1}{\operatorname{Re}} \nabla \cdot \boldsymbol{\tau}=\beta C n^{2} \rho \nabla \nabla^{2} \rho,
\end{aligned}
$$

$$
p=\frac{\rho T}{(1-\rho / 3)}-\frac{9}{8} \rho^{2}
$$

As mentioned above, these equations are based on a convective scaling and therefore they become unstable at the very early stages of the process, when phase separation is dominated by diffusion. Therefore, the early stages were simulated using the equations based on the diffusive scaling, Eqs. (12) and (13).

Again, we stress that all external forces, and buoyancy in particular, are assumed to be negligible. That means that drop and bubble sizes $\ell$ are small compared to the capillary length, i.e., $\ell \ll \sqrt{\sigma / g(\Delta \rho)_{\text {eq }}}$, where $g$ is the gravity field, which amounts to assuming that the Bond number is very small. Also, for the sake of simplicity, in the following we will assume $\beta=1$.

\section{NUMERICAL RESULTS}

Numerical methods here are based on previous works by Nagarajan et al. ${ }^{34,35}$ In their methodology, the compressible Navier-Stokes equations for an inert calorically perfect gas are discretized in conservative form using a structured, staggered arrangement of the conserved variables, with spatial derivatives computed via sixth-order compact finite differences. ${ }^{36}$ Combined with an explicit Runge-Kutta or an implicit/explicit temporal scheme, this numerical method has been extensively tested ${ }^{34}$ and successfully employed for simulating bypass transition in a boundary layer. ${ }^{35}$ Based on the same compact finite-difference discretization on staggered grids, the governing equations (16)-(18) are integrated in a $2 \mathrm{D}$ or $3 \mathrm{D}$ periodic box with temporal advancement effected via a third-order Runge-Kutta scheme.

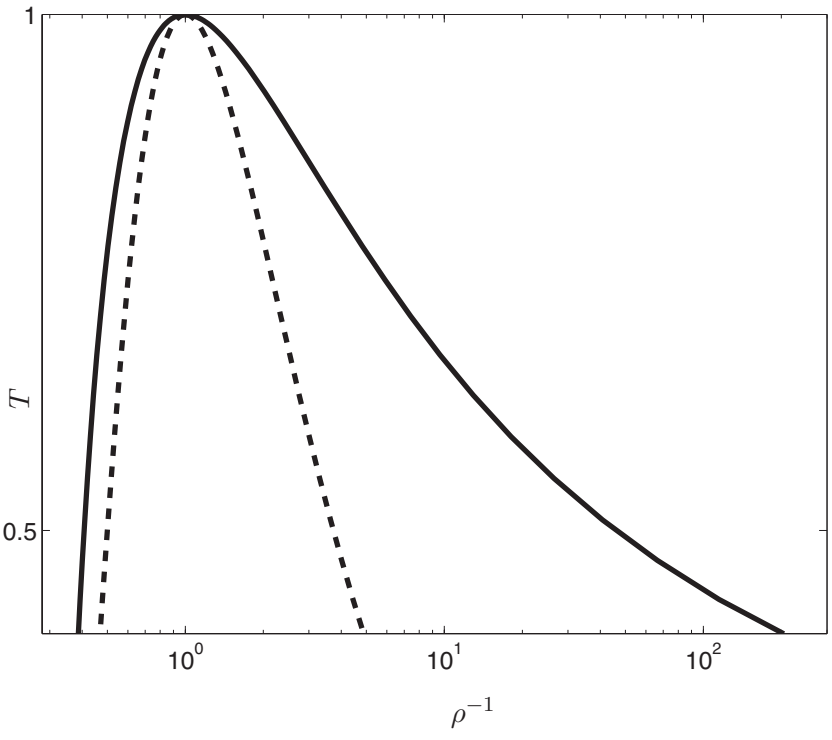

FIG. 1. Binodal and spinodal curves for a van der Waals fluid. Requiring that at any temperature the two phases have equal pressure, temperature, and chemical potential yields the binodal. Solving $(\partial p / \partial \rho)_{T}=0$ [with $p$ given by Eq. (11)] determines the spinodal.

\section{A. Binary droplet coalescence in vapor at equilibrium}

We simulate the coalescence process of two identical liquid droplets that are at equilibrium with a continuous vapor phase in two dimensions. Assuming a homogeneous and constant temperature field, $T=0.9$, from Fig. 1 we see that the corresponding densities of the two phases are $\rho_{\mathrm{I}}=1.65$ and $\rho_{\mathrm{II}}=0.425$. Initially, the liquid droplets are quiescent, with radius $4 a$ and are placed $10 a$ apart from each other, so that they "touch." We expect that since the Korteweg force tends to minimize surface energy, it will result in a nonequilibrium attractive force between the two drops, inducing coalescence. In fact, as can be seen in Fig. 2, the droplets are attracted to each other and merge. Then, they start to oscillate due to surface undulations at the interface after the merger. The period of oscillation and droplet radii were re-
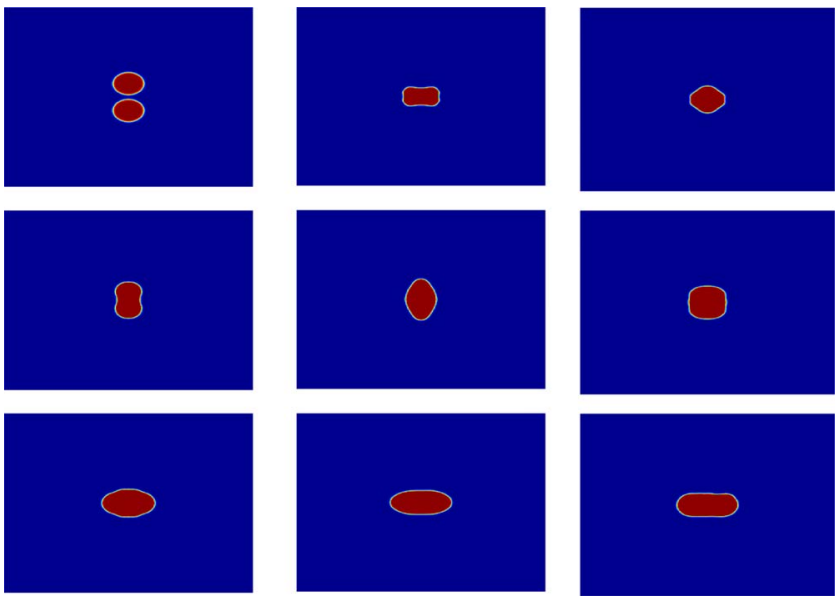

FIG. 2. (Color online) Snapshots of near-critical droplet coalescence at different nondimensional times (diffusive scaling) $10^{3} t=0,8,9,10,11,12,13$, 14 , and 15 (left to right and top to bottom) for $\mathcal{R}=1000$. 

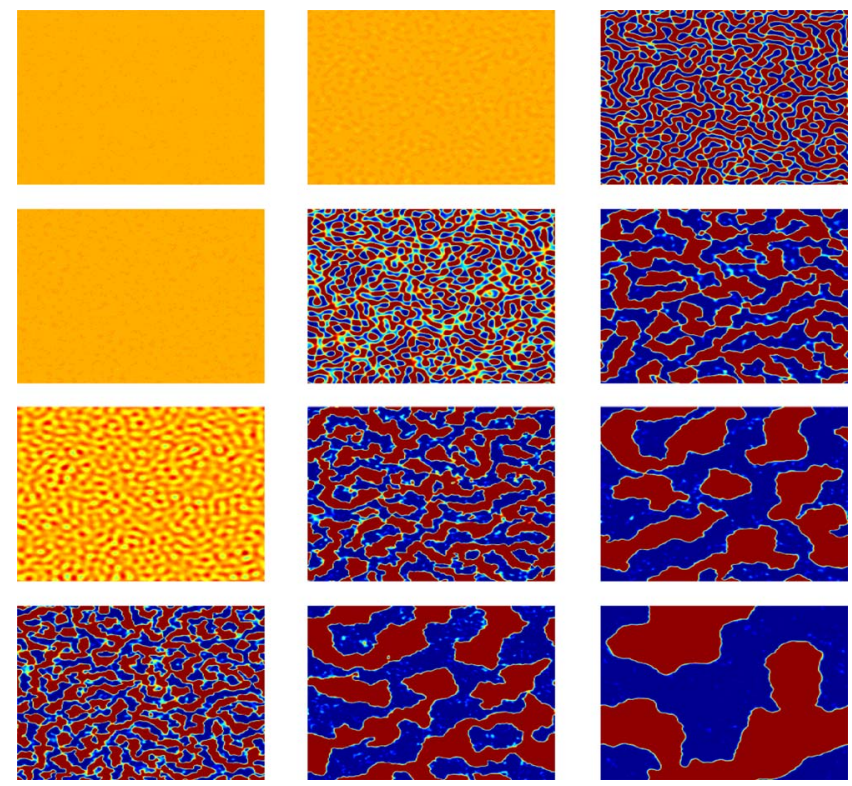

FIG. 3. (Color online) Liquid-vapor spinodal decomposition of a critical van der Waals fluid (with $\rho_{0}=1.042$ ) at different nondimensional times (diffusive scaling) $t=10^{-3}, 5 \times 10^{-3}$, and $2 \times 10^{-2}$, from $2 \mathrm{D}$ simulations with $256^{2}$ grid points with $\mathcal{R}=1,10,10^{2}$, and $10^{3}$ from top to bottom.

corded and found consistent with the relation for capillary waves at a cylindrical liquid-vapor interface ${ }^{37} \omega^{2} \sim R^{-3}$.

\section{B. Liquid-vapor spinodal decomposition}

We investigate liquid-vapor spinodal decomposition of a van der Waals fluid which is instantaneously quenched into the unstable region of its phase diagram (i.e., under its liquid-vapor coexistence curve). The simulations are conducted at $T=0.9$, for negligible Bond number and for different values of $\mathcal{R}$, assuming an infinite expanse of fluid, which is thus modeled via periodic boundary conditions. At this temperature, a representative vapor-liquid viscosity ratio is chosen to be $r=10^{-3}$. Initially, we assume quiescent conditions and a density field being the sum of a Gaussian white noise superposed on a uniform constant density $\rho=\rho_{0}$. We chose values of $\rho_{0}=0.7,1.042,1.3$ in the spinodal range, i.e., $\rho_{0} \in\left[\rho_{s}^{\mathrm{II}}, \rho_{s}^{\mathrm{I}}\right]$, where $\rho_{s}^{\mathrm{I}}=1.39$ and $\rho_{s}^{\mathrm{II}}=0.654$ denote the liquid and vapor spinodal densities at the given temperature. [For a van der Waals fluid these values are easily found by solving the algebraic relation $\rho(3-\rho)^{2}=4 T$, plotted as the dashed line in Fig. 1.] Specifically, $\rho_{0}=1.042$ is the critical density, while $\rho_{0}=0.7$ and $\rho_{0}=1.3$ correspond to vapor-rich and liquid-rich mixtures, respectively. As expected, after the critical quench $\rho_{0}=1.042$, the phase-ordering process (Fig. 3) is characterized by the formation of bicontinuous structures, which subsequently grow and coalesce. For the off-critical quenches $\rho_{0}=0.7$ (Fig. 4) and $\rho_{0}=1.3$ (Fig. 5), the phase separation pattern consists of a random collection of rapidly coalescing pseudospherical nuclei of the minority phase, surrounded by the majority phase. As can be seen, only after the first spinodal pattern is formed (i.e., a bicontinuous pattern for the critical quench, or a random collection of nuclei for the offcritical quenches), do the single-phase domains start to grow and coalesce, at an increasing rate for larger $\mathcal{R}$.
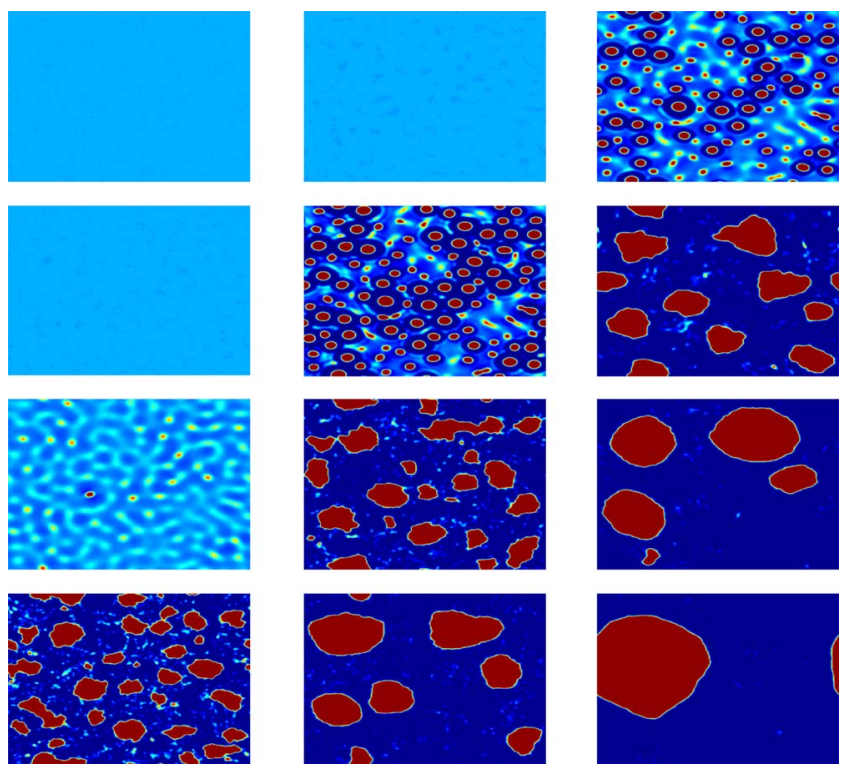

FIG. 4. (Color online) Liquid-vapor spinodal decomposition of an offcritical van der Waals fluid (with $\rho_{0}=0.7$ ) at different nondimensional times (diffusive scaling) $t=5 \times 10^{-3}, 2 \times 10^{-2}$, and $1 \times 10^{-1}$, from $2 \mathrm{D}$ simulations with $256^{2}$ grid points with $\mathcal{R}=1,10,10^{2}$, and $10^{3}$ from top to bottom.

As a quantitative characterization of the influence of the convection parameter $\mathcal{R}$ on the average phase composition of the two-phase fluid, we define the separation depth, $s$, measuring the "distance" of the single-phase domains from their equilibrium state, ${ }^{38}$ as

$$
s=\left\langle\frac{\rho(x)-\rho_{0}}{\rho_{\mathrm{eq}}(\boldsymbol{x})-\rho_{0}}\right\rangle,
$$

where $\rho_{0}$ is the initial mean density, and the brackets indicate volume and ensemble averaging. Here, $\rho_{\mathrm{eq}}$ denotes the
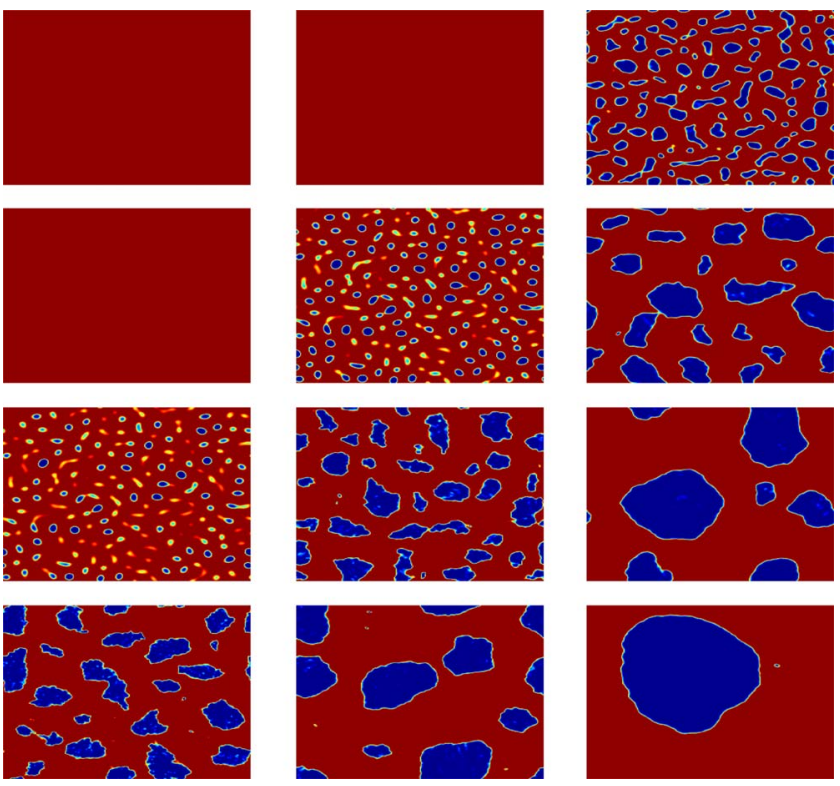

FIG. 5. (Color online) Liquid-vapor spinodal decomposition of an offcritical van der Waals fluid (with $\rho_{0}=1.3$ ) at different nondimensional times (diffusive scaling) $t=3 \times 10^{-3}, 1 \times 10^{-2}$ and $5 \times 10^{-2}$, from $2 \mathrm{D}$ simulations with $256^{2}$ grid points with $\mathcal{R}=1,10,10^{2}$, and $10^{3}$ from top to bottom. 


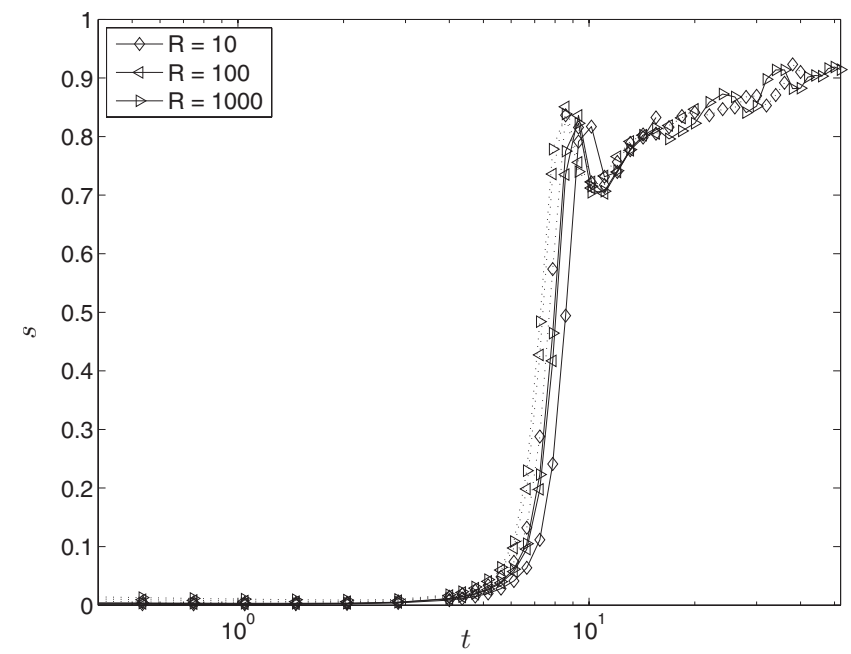

FIG. 6. Separation depth vs time (acoustic scaling) for different values of $\mathcal{R}=10,100,1000$ from 2D simulations with $\rho_{0}=0.7$ (solid curve) vs 3D simulation results (dotted curve).

steady-state density of the liquid phase, $\rho_{\mathrm{I}}$, or of the vapor phase, $\rho_{\mathrm{II}}$, depending on the local density $\rho(\boldsymbol{x})$, i.e.,

$$
\rho_{\mathrm{eq}}(x)= \begin{cases}\rho_{\mathrm{I}}, & \text { if } \rho(x)>\rho_{0}, \\ \rho_{\mathrm{II}}, & \text { if } \rho(x)<\rho_{0} .\end{cases}
$$

Figures 6-8 show the temporal evolution (in acoustic time units) of the separation depth for the critical and off-critical quenches for $\mathcal{R}=1,10,100,1000$. The solid curves in these figures were obtained from 2D simulations on a $256^{2}$ grid, whereas the dotted lines are from 3D simulations on a $128^{3}$ grid. Identical results were obtained using $512^{2}$ and $256^{3}$ grids; in fact, as shown in Fig. 9, the same curve for the separation depth was obtained using $128^{2}, 256^{2}$, and $512^{2}$ grids, and analogously for the 3D case. Figures 6-8 show remarkable quantitative agreement between the solid and dotted sets of curves. For large times, to quite good accuracy, the curves for different values of $\mathcal{R}$ can be collapsed to a single curve, suggesting that the temporal evolution in the

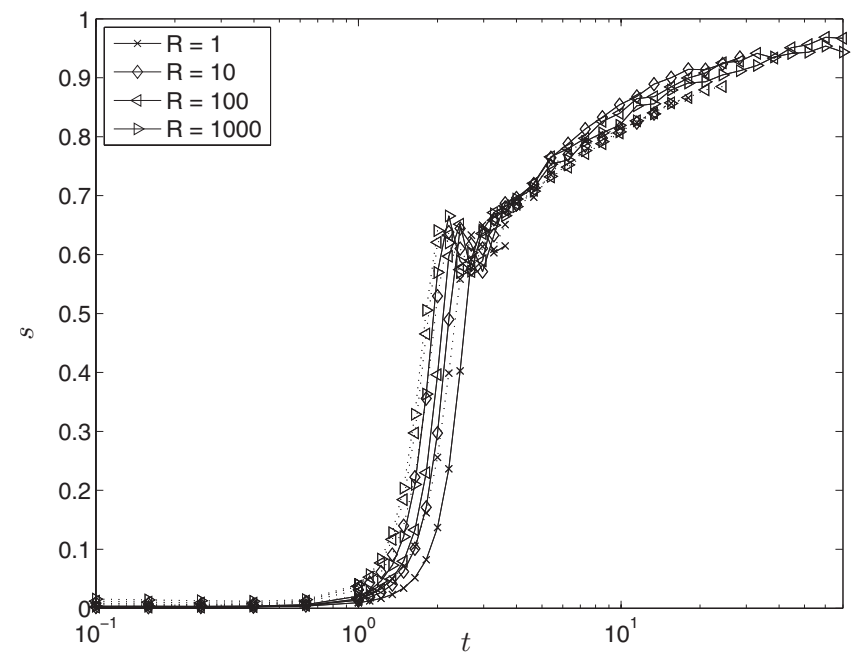

FIG. 7. Separation depth vs time (acoustic scaling) for different values of $\mathcal{R}=1,10,100,1000$ from 2D simulations with $\rho_{0}=1.042$ (solid curve) vs 3D simulation results (dotted curve).

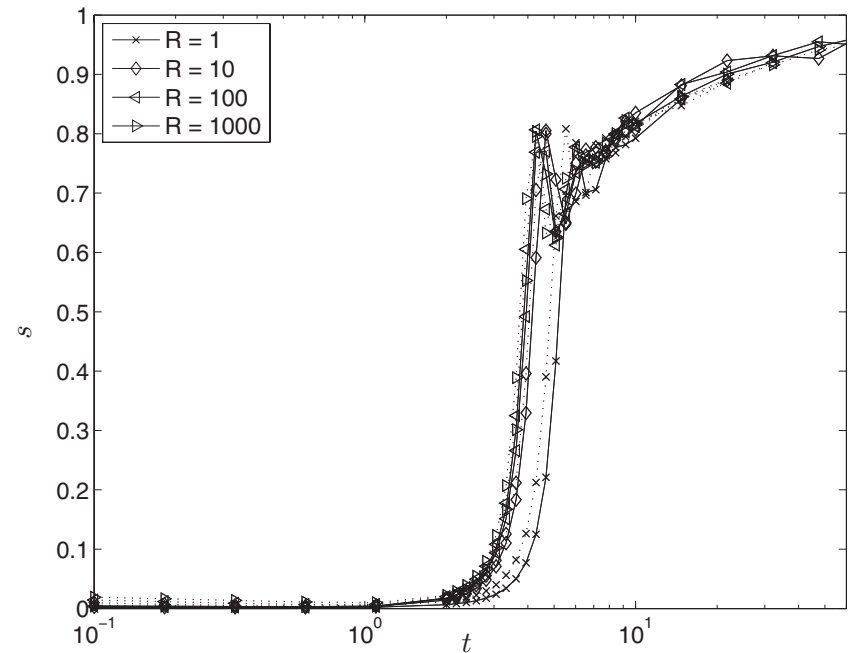

FIG. 8. Separation depth vs time (acoustic scaling) for different values of $\mathcal{R}=1,10,100,1000$ from 2D simulations with $\rho_{0}=1.3$ (solid curve) vs 3D simulation results (dotted curve).

inertial regime is self-similar. All curves show that the process takes place in three steps. First, there is a time delay, when no detectable phase separation takes place. As noted by Vladimirova et al. ${ }^{38}$ this delay depends on the depth of the temperature quench and the intensity of the random noise, while it is almost independent of $\mathcal{R}$. Then, at the onset of phase separation, the process is very rapid and the separation depth "jumps" to a value close to one, independently of the value of $\mathcal{R}$, showing that the system reaches local equilibrium shortly after sharp interfaces are formed. Afterwards, in the third stage, phase separation proceeds much more slowly, as the density gradients within the single-phase domains are very small, while the densities of the two phases across any interface change only very slowly in time, asymptotically approaching equilibrium at $s=1$.

Since the Korteweg force is nondissipative, the total energy of the system decreases due to viscous forces only, i.e.,

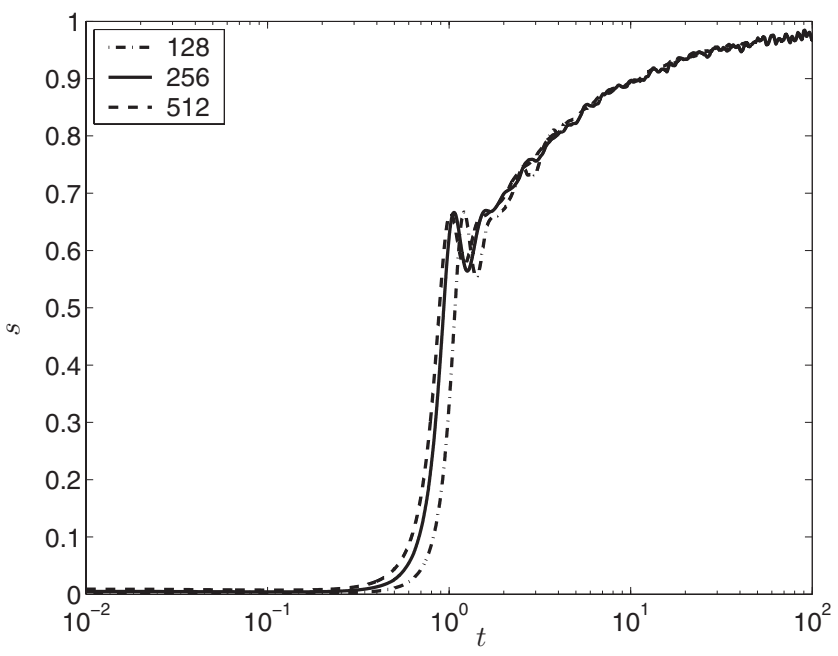

FIG. 9. Separation depth vs time (acoustic scaling) for $\rho_{0}=1.042$ and $\mathcal{R}$ $=1000$ from $2 \mathrm{D}$ simulations with $128^{2}, 256^{2}, 512^{2}$ grid points. 


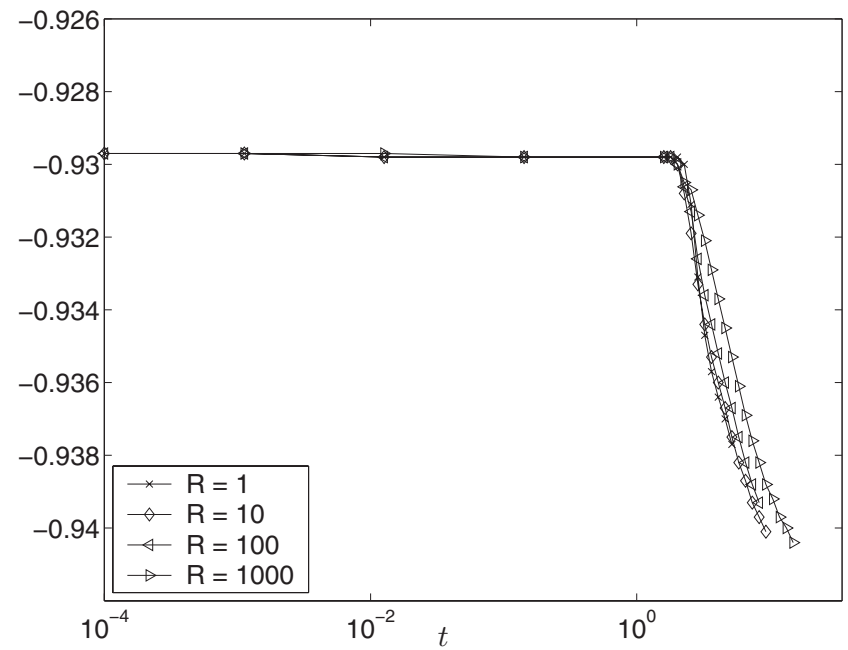

FIG. 10. Mean total energy vs time (acoustic scaling) for different values of $\mathcal{R}=1,10,100,1000$ with $\rho_{0}=1.3$ from $2 \mathrm{D}$ simulations with $256^{2}$ grid points.

$$
\frac{d}{d t}\left\langle\frac{1}{2} \rho \mathbf{u}^{2}+f\right\rangle=-2\left\langle\eta|\dot{\mathbf{D}}|^{2}\right\rangle,
$$

where $\mathbf{\mathbf { D }}$ is the traceless component of the rate of strain tensor and the brackets denote a space average. This fact was checked in Fig. 10, which shows the mean total energy [as defined by the bracketed quantity on the left-hand side of Eq. (21)] from $2 \mathrm{D}$ simulations on a $256^{2}$ grid. Here we see that dissipation starts to occur only after microdomains have appeared, i.e., when viscous forces become relevant, while at earlier times mechanical energy is conserved. This confirms that dissipation is due exclusively to viscous forces, as the velocity field induced by the Korteweg stresses is potential.

Next, we studied the rate of coarsening as reflected in the growth law for the integral scale of the radial paircorrelation function,

$$
\mathcal{C}(r, t)=\frac{1}{2 \pi \rho_{\mathrm{rms}}^{2}} \int\langle\widetilde{\rho}(\mathbf{x}+\mathbf{r}) \widetilde{\rho}(\mathbf{x})\rangle d \theta,
$$

which can re-expressed as

$$
\mathcal{L}(t)=\frac{1}{\rho_{\text {rms }}^{2}} \sum_{\mathbf{k}} \frac{\left\langle\left|\hat{\tilde{\rho}}_{\mathbf{k}}\right|^{2}\right\rangle}{|\mathbf{k}|}
$$

where $\tilde{\rho}=\rho-\langle\rho\rangle, \rho_{\text {rms }}$ is the root-mean-square value of $\rho$, hats denote Fourier transforms, while the brackets denote averaging over a shell in Fourier space at fixed $k=|\mathbf{k}|$.

The prediction of scaling theory ${ }^{21,22}$ (setting aside the caveat in Sec. I) is that during the late stage of phase separation,

$$
\mathcal{L}=\mathcal{A} t^{2 / 3},
$$

where $\mathcal{A}$ denotes the (dimensionless) $(\sigma / \rho)^{1 / 3}$ ratio, i.e.,

$$
\mathcal{A} \equiv\left(\frac{a^{2} \beta T \int(\nabla \rho)^{2} d \ell}{\rho_{r}}\right)^{1 / 3},
$$

with $\rho_{r}$ denoting a reference density (all quantities being dimensionless within the acoustic scaling). As can be seen in Figs. 11-13, during the first stage of the process the rate of

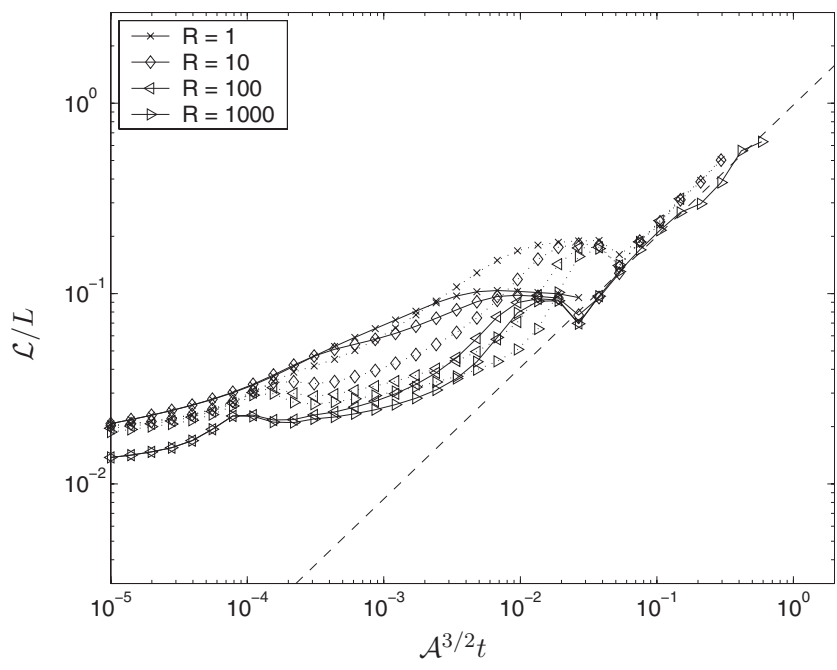

FIG. 11. Integral scale vs time (acoustic scaling) for different values of $\mathcal{R}$ $=1,10,100,1000$ from $2 \mathrm{D}$ simulations with $\rho_{0}=0.7$ (solid curve) vs $3 \mathrm{D}$ simulation results (dotted curve). Also shown (dashed curve) is a $\frac{2}{3}$ slope line.

coarsening is strongly influenced by the chosen value for the convection parameter, until sharp interfaces are formed. Then, domains stop growing, concomitant to their composition rapidly reaching local equilibrium. Finally, during the latest stage, growth is driven by inertial forces and is characterized by a $\frac{2}{3}$ power-law behavior, in agreement with Eq. (24). In fact, when we plot $\mathcal{L} t^{-2 / 3}$ versus $t$ for both $2 \mathrm{D}$ and $3 \mathrm{D}$ simulations, we see that the curves tend to the same asymptotic value at large times, i.e., a constant value of $\mathcal{A}$, independent of $\mathcal{R}$. Considering that $\mathcal{A} \propto(\sigma / \rho)^{1 / 3}$, this confirms that each data set with a prescribed value of $\langle\rho\rangle$ has the same (dimensionless) surface tension. We also checked that the value of $\mathcal{A}$ that we obtained agrees within 20\% accuracy with Eq. (25).

Similar behavior is shown by the reciprocal first moment of the spherically averaged structure factor, given by

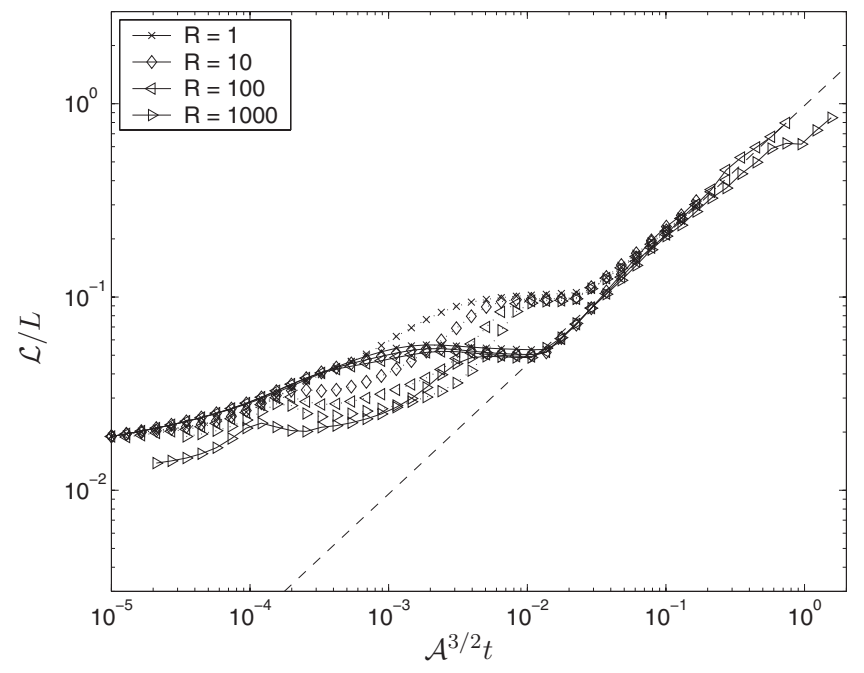

FIG. 12. Integral scale vs time (acoustic scaling) for different values of $\mathcal{R}$ $=1,10,100,1000$ from 2D simulations with $\rho_{0}=1.042$ (solid curve) vs 3D simulation results (dotted curve). Also shown (dashed curve) is a $\frac{2}{3}$ slope line. 


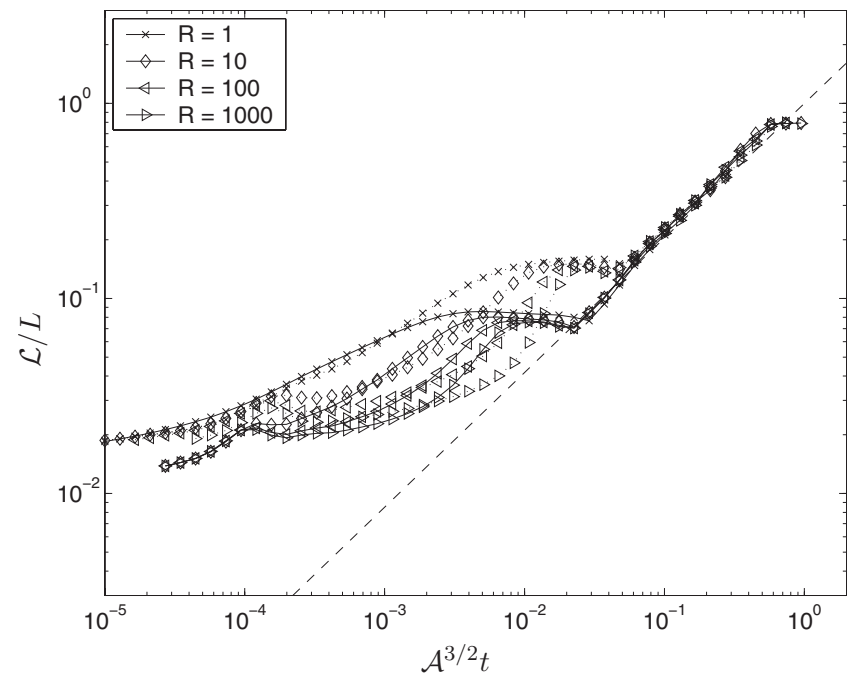

FIG. 13. Integral scale vs time (acoustic scaling) for different values of $\mathcal{R}$ $=1,10,100,1000$ from $2 \mathrm{D}$ simulations with $\rho_{0}=1.3$ (solid curve) vs $3 \mathrm{D}$ simulation results (dotted curve). Also shown (dashed curve) is a $\frac{2}{3}$ slope line.

$$
\Lambda(t)=\frac{\Sigma_{\mathbf{k}}\left\langle\left|\hat{\tilde{\rho}}_{\mathbf{k}}\right|^{2}\right\rangle}{\Sigma_{\mathbf{k}}|\mathbf{k}|\left\langle\left|\hat{\tilde{\rho}}_{\mathbf{k}}\right|^{2}\right\rangle} .
$$

Finally, as a measure of the strength of the fluid motion induced by phase segregation, we define a Reynolds number based on the root-mean-square velocity, $u_{\text {rms }}$, and the characteristic size of single-phase microdomains, $\mathcal{L}$, i.e., $\mathfrak{R}$ $=\rho_{c} u_{\mathrm{rms}} \mathcal{L} / \eta_{\mathrm{I}}$. In Figs. $14-16$, this quantity is plotted as a function of $\mathcal{R}$. At first glance these plots would suggest that the temporal evolution is self-similar. However, close inspection reveals that, particularly at large times, once the curves are stripped bare of $\operatorname{Re}$ (i.e., of the $\mathcal{R}^{1 / 2}$ dependence) they do not collapse on a single curve but, in fact, show a dependence on $\mathcal{R}$, albeit very weak. However, this dependence, together with the asymptotic behavior of $\mathfrak{R}$ at large times, cannot be reliably addressed using the present data set, due

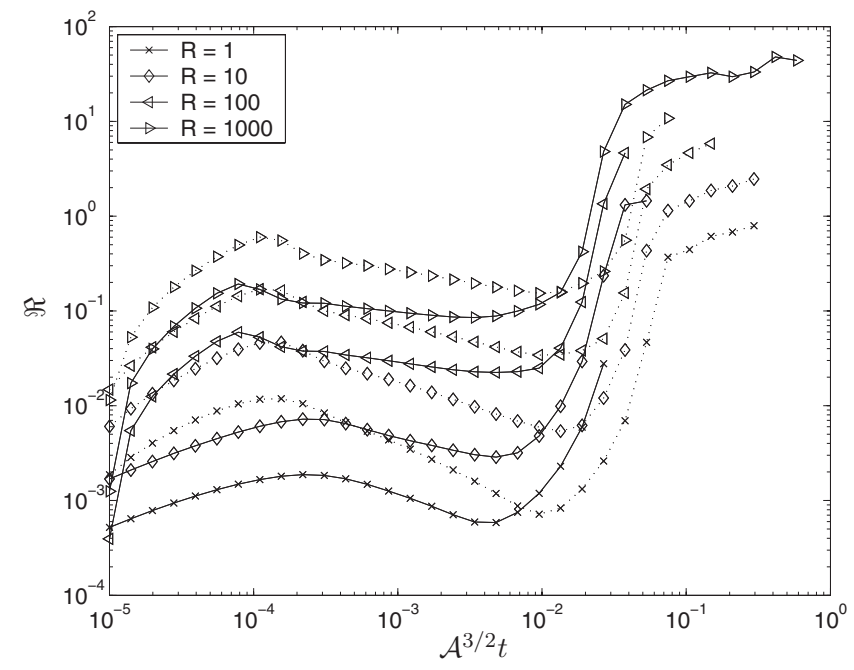

FIG. 14. Reynolds number $\mathfrak{R}$ vs time (acoustic time units) for different values of $\mathcal{R}=1,10,100,1000$ from $2 \mathrm{D}$ simulations with $\rho_{0}=0.7$ (solid curve) vs 3D simulation results (dotted curve).

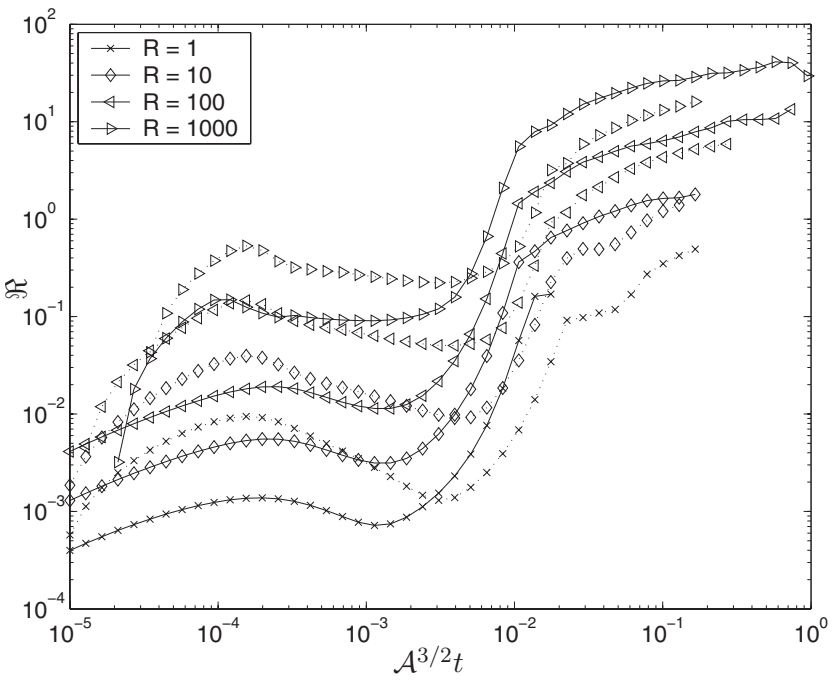

FIG. 15. Reynolds number $\mathfrak{R}$ vs time (acoustic time units) for different values of $\mathcal{R}=1,10,100,1000$ from $2 \mathrm{D}$ simulations with $\rho_{0}=1.042$ (solid curve) vs $3 \mathrm{D}$ simulation results (dotted curve).

to finite box size effects which spoil the scaling laws at large times, and will be addressed using higher resolution simulations.

\section{DISCUSSION AND CONCLUSIONS}

The objective of this work is to simulate spinodal decomposition of a van der Waals fluid, following the diffuseinterface model. First, we developed the equations of motion rigorously, coupling the van der Waals equation of state and the equations of conservation of mass, momentum, and energy with basic principles of irreversible thermodynamics. Then, we restricted our attention to cases where (a) drop and bubble sizes are much smaller than the capillary length, so that buoyancy forces can be neglected; (b) phase separation occurs isothermally so that energy is identically conserved.

In these conditions, phase separation appears to take place in three steps. In the first stage, the system fluctuates,

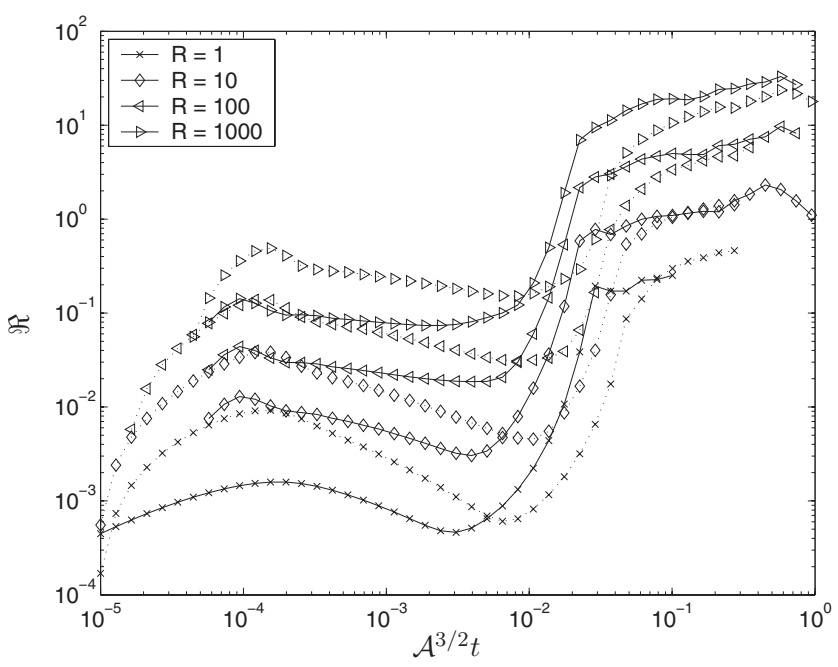

FIG. 16. Reynolds number $\mathfrak{R}$ vs time (acoustic time units) for different values of $\mathcal{R}=1,10,100,1000$ from $2 \mathrm{D}$ simulations with $\rho_{0}=1.3$ (solid curve) vs 3D simulation results (dotted curve). 
with a typical length scale growing with time, but with no detectable phase separation taking place. Accordingly, the onset of phase separation is delayed, in much the same way that we have observed for liquid, viscous binary mixtures. ${ }^{38,39}$ In the second stage, sharp interfaces are formed around nuclei that hardly grow in time, but whose composition rapidly reaches local equilibrium. Finally, in the third stage, these nuclei at local equilibrium start to grow in time like $t^{2 / 3}$, showing that capillary forces are balanced by inertial forces.

So, during the second and third stages of liquid-vapor phase separation for a van der Waals fluid, first the system reaches local equilibrium (with the formation of nuclei having sharp interfaces), and then these nuclei start to grow. This is in contrast with phase separation in viscous liquid binary mixtures, where the two events occur simultaneously. In fact, for viscous binary mixtures, after the (delayed) onset of phase separation, local equilibrium is reached well after the appearance of nuclei with sharp interfaces, through a process of composition relaxation of the nuclei that is concomitant to their temporal growth. In addition, in liquid-liquid phase separation, capillary forces are balanced by viscous forces, leading to a linear growth law of the nuclei sizes, while for liquid-vapor phase transition, capillary forces are balanced by inertial forces, leading to a $2 / 3$ power-law behavior.

\section{ACKNOWLEDGMENTS}

Support and computational resources made available by the Center for Turbulence Research, Stanford University, are gratefully acknowledged.

${ }^{1}$ R. Gupta, R. Mauri, and R. Shinnar, "Phase separation of liquid mixtures in the presence of surfactants," Ind. Eng. Chem. Res. 38, 2418 (1999).

${ }^{2}$ R. Mauri, F. Califano, E. Calvi, R. Gupta, and R. Shinnar, "Convectiondriven phase segregation of deeply quenched liquid mixtures," J. Chem. Phys. 118, 8841 (2003).

${ }^{3}$ F. Califano and R. Mauri, "Drop size evolution during the phase separation of liquid mixtures," Ind. Eng. Chem. Res. 43, 349 (2004).

${ }^{4}$ P. Poesio, G. Cominardi, A. Lezzi, R. Mauri, and G. P. Beretta, "Effects of quenching rate and viscosity on spinodal decomposition," Phys. Rev. E 74, 011507 (2006).

${ }^{5}$ N. Vladimirova, A. Malagoli, and R. Mauri, "Diffusiophoresis of twodimensional liquid droplets in a phase-separating system," Phys. Rev. E 60, 2037 (1999).

${ }^{6}$ W. R. Osborn, E. Orlandini, M. R. Swift, J. M. Yeomans, and J. R. Banavar, "Lattice Boltzmann study of hydrodynamic spinodal decomposition," Phys. Rev. Lett. 75, 4031 (1995).

${ }^{7}$ R. Yamamoto and K. Nakanishi, "Computer simulation of vapor-liquid phase separation," Mol. Simul. 16, 119 (1996).

${ }^{8} \mathrm{P}$. B. Warren, "Hydrodynamic bubble coarsening in off-critical vaporliquid phase separation," Phys. Rev. Lett. 87, 225702 (2001).

${ }^{9}$ D. Beysens, Y. Garrabos, V. S. Nikolayev, C. Lecoutre-Chabot, J.-P. Delville, and J. Hegseth, "Liquid-vapor phase separation in a thermocapillary force field," Europhys. Lett. 59, 245 (2002).

${ }^{10}$ V. Sofonea, A. Lamura, G. Gonnella, and A. Cristea, "Finite-difference lattice Boltzmann model with flux limiters for liquid-vapor systems," Phys. Rev. E 70, 046702 (2004).
${ }^{11}$ R. Borcia and M. Bestehorn, "Phase-field simulations for drops and bubbles," Phys. Rev. E 75, 056309 (2007).

${ }^{12}$ A. Oprisan, S. A. Oprisan, J. Hegseth, Y. Garrabos, C. Lecoutre-Chabot, and D. Beysens, "Universality in early-stage growth of phase-separating domains near the critical point," Phys. Rev. E 77, 051118 (2008).

${ }^{13}$ B. U. Felderhof, "Dynamics of the diffuse gas-liquid interface near the critical point," Physica (Amsterdam) 48, 541 (1970).

${ }^{14}$ J. S. Langer and L. A. Turski, "Hydrodynamic model of the condensation of a vapor near its critical point," Phys. Rev. A 8, 3230 (1973).

${ }^{15}$ D. D. Joseph, "Fluid dynamics of two miscible liquids with diffusion and gradient stresses," Eur. J. Mech. B/Fluids 6, 565 (1990).

${ }^{16}$ L. K. Antanovskii, "Microscale theory of surface tension," Phys. Rev. E 54, 6285 (1996).

${ }^{17}$ D. Jasnow and J. Viñals, "Coarse-grained description of thermo-capillary flow," Phys. Fluids 8, 660 (1996).

${ }^{18}$ J. Lowengrub and L. Truskinovsky, "Quasi-incompressible Cahn-Hilliard fluids and topological transitions," Proc. R. Soc. London, Ser. A 454, 2617 (1998).

${ }^{19}$ D. M. Anderson, G. B. McFadden, and A. A. Wheeler, "Diffuse-interface methods in fluid mechanics," Annu. Rev. Fluid Mech. 30, 139 (1998).

${ }^{20}$ V. E. Badalassi, H. D. Ceniceros, and S. Banerjee, "Computation of multiphase systems with phase field models," J. Comput. Phys. 190, 371 (2003).

${ }^{21}$ E. Siggia, "Late stages of spinodal decomposition in binary mixtures," Phys. Rev. A 20, 595 (1979).

${ }^{22} \mathrm{H}$. Furukawa, "Role of inertia in the late stage of the phase separation of a fluid," Physica A 204, 237 (1994).

${ }^{23}$ C. Appert, J. F. Olson, D. H. Rothman, and S. Zaleski, "Phase separation in a three-dimensional, two-phase, hydrodynamic lattice gas," J. Stat. Phys. 81, 181 (1995).

${ }^{24}$ V. M. Kendon, M. E. Cates, I. Pagonabarraga, J.-C. Desplat, and P. Bladon, "Inertial effects in three-dimensional spinodal decomposition of a symmetric binary fluid mixture," J. Fluid Mech. 440, 147 (2001).

${ }^{25}$ J. Chin and P. V. Coveney, "Lattice Boltzmann study of spinodal decomposition in two dimensions," Phys. Rev. E 66, 016303 (2002).

${ }^{26}$ M. Grant and K. R. Elder, "Spinodal decomposition in fluids," Phys. Rev. Lett. 82, 14 (1999).

${ }^{27}$ V. M. Kendon, "Scaling theory of three-dimensional spinodal turbulence," Phys. Rev. E 61, R6071 (2000).

${ }^{28}$ J. S. Rowlinson and B. Widom, Molecular Theory of Capillarity (Dover, New York, 2002), Chap. 3.

${ }^{29}$ L. M. Pismen, "Non-local diffuse-interface theory of thin films and moving contact line," Phys. Rev. E 64, 021603 (2001).

${ }^{30}$ D. Molin and R. Mauri, "Enhanced heat transport during phase separation of liquid binary mixtures," Phys. Fluids 19, 074102 (2007).

${ }^{31}$ J. D. van der Waals, "The thermodynamic theory of capillarity under the hypothesis of a continuous variation of density," Z. Phys. Chem. Stoechiom. Verwandtschaftsl. 13, 657 (1894) [translated and reprinted in J. Stat. Phys. 20, 200 (1979)].

${ }^{32}$ S. R. de Groot and P. Mazur, Non-Equilibrium Thermodynamics (Dover, New York, 1984).

${ }^{33}$ D. Jacqmin, "Contact-line dynamics of a diffuse fluid interface," J. Fluid Mech. 402, 57 (2000).

${ }^{34}$ S. Nagarajan, S. K. Lele, and J. H. Ferziger, "A robust high-order compact method for large-eddy simulation,” J. Comput. Phys. 191, 392 (2003).

${ }^{35}$ S. Nagarajan, S. K. Lele, and J. H. Ferziger, "Leading-edge effects in bypass transition," J. Fluid Mech. 572, 471 (2007).

${ }^{36}$ S. K. Lele, "Compact finite-difference schemes with spectral-like resolution," J. Comput. Phys. 103, 16 (1992).

${ }^{37}$ H. Lamb, Hydrodynamics, 6th ed. (Dover, New York, 1993), Chap. 9.

${ }^{38} \mathrm{~N}$. Vladimirova, A. Malagoli, and R. Mauri, "Diffusion-driven phase separation of deeply quenched mixtures," Phys. Rev. E 58, 7691 (1998).

${ }^{39} \mathrm{~N}$. Vladimirova, A. Malagoli, and R. Mauri, "Two-dimensional model of phase segregation in liquid binary mixtures," Phys. Rev. E 60, 6968 (1999). 